\title{
Analysis of flow observables in small systems using an inte- grated dynamical model
}

\author{
Koji Kawaguchi ${ }^{1, \star}$, Koichi Murase ${ }^{2,1, \star \star}$, and Tetsufumi Hirano ${ }^{1, \star \star \star}$ \\ ${ }^{1}$ Department of Physics, Sophia University, Tokyo 102-8554, Japan \\ ${ }^{2}$ Department of Physics, The University of Tokyo, Tokyo 113-0033, Japan
}

\begin{abstract}
We investigate collectivity in small colliding systems by using an integrated dynamical model in which Monte-Carlo initialisation of hydrodynamic fields, the ideal hydrodynamic description of the quark-gluon plasma and the kinetic description of the hadron gas are incorporated. We implement fluctuations of the multiplicity and of the longitudinal matter profile in the initial conditions which are of particular importance in small colliding systems. We also discuss the effects of hadronic rescatterings on flow observables.
\end{abstract}

\section{Introduction}

The perfect fluidity of the quark-gluon plasma (QGP) created in high energy nuclear collisions was discovered at Relativistic Heavy Ion Collider (RHIC) [1]. The perfect-fluid-like collective behaviour of the QGP is well described by relativistic hydrodynamics. One of the main observables to gain information of the bulk and the transport properties of the QGP is the second harmonics of azimuthal angle distributions, the so-called elliptic flow [2].

Since the system size dependence of the observables is one of the keys to get information about the property of the matter, small colliding systems attract a great deal of attention. Collective flow in small colliding systems had not been studied since the system is not supposed to reach thermal equilibrium in these systems. However, recent measurements reveal that the system exhibits collective-flow-like behaviour even in $p / d{ }^{3} \mathrm{He}-\mathrm{Au}$ collisions at RHIC $[3,4]$. All the low $p_{\mathrm{T}}$ data in small colliding systems are consistent with a hydrodynamic flow picture [5]. Thus it would be interesting to see whether flow observables really result from the presence of the QGP. Therefore we analyse flow observables in small colliding systems at the RHIC energy by employing an integrated dynamical model based on an assumption of QGP fluid formation.

\section{Model}

In order to describe the dynamics of high energy nuclear collisions at the RHIC energy, we employ an integrated dynamical model [6] which is combination of Monte-Carlo Glauber model (MC-Glauber)

\footnotetext{
^e-mail: kawaguchi@eagle.sophia.ac.jp

$\star \star$ e-mail: murase@nt.phys.s.u-tokyo.ac.jp

$\star \star \star$ e-mail: hirano@sophia.ac.jp
} 
for the initial entropy production, ideal hydrodynamics for QGP fluids in the intermediate stage and a hadronic cascade model for hadron gases in the late stage. In the initial stage, we use MC-Glauber to calculate initial entropy density distributions just after collisions of two nuclei. Space-time evolution of the QGP is described by fully $(3+1)$ dimensional ideal hydrodynamic simulations. After that we switch from fluids to hadron gases with the Cooper-Frye formula [7] in the isothermal hypersurface at the temperature of $155 \mathrm{MeV}$. For the evolution of the hadronic gas, we use a hadron cascade code, JAM [8].

In small colliding systems, one needs to extend a model of initial conditions in hydrodynamic simulations by taking account of event-by-event fluctuations of geometry and initial entropy densities. Since an initial entropy density profile is parametrised as a smooth function in $p+p$ collisions in the original integrated dynamical model, final multiplicity fluctuations cannot be reproduced in $p+p$ collisions. To resolve this problem, we employ an event generator, PYTHIA [9], which naturally describes multiplicity distributions in elementary $p+p$ collisions. For each pair of binary collisions, we run PYTHIA for one inelastic $p+p$ collisions. For the soft particle production, it is well known that multiplicities scale with the number of participants, rather than the number of binary collisions. To account for this, we perform a rejection sampling from final particle lists in PYTHIA for pairs of all binary collisions so that the multiplicities scale with the number of participants for a given rapidity. By taking into account an idea of "rapidity triangle/trapezoid" [10-12], we parametrise the rapidity-dependent acceptance probability of the rejection sampling as

$$
w(Y)=\frac{Y_{\mathrm{b}}+Y}{2 Y_{\mathrm{b}}} \frac{1}{N_{\mathrm{A}}}+\frac{Y_{\mathrm{b}}-Y}{2 Y_{\mathrm{b}}} \frac{1}{N_{\mathrm{B}}}
$$

where $Y$ is rapidity and $Y_{\mathrm{b}}$ is beam rapidity. $N_{\mathrm{A}}$ and $N_{\mathrm{B}}$ are the number of nucleons in a nucleus $\mathrm{A}$ and $\mathrm{B}$, respectively. We assume that the initial entropy density distribution is proportional to the number distribution of produced particles and that each produced particle is associated with a Gaussian function in the configuration space. Thus the entropy density distribution at initial proper time $\tau_{0}$ in full three-dimensional space is

$$
s\left(\tau_{0}, x, y, \eta_{\mathrm{s}}\right)=\frac{K}{\tau_{0}} \sum_{i} \frac{1}{\sqrt{2 \pi \sigma_{\eta}^{2}}} \frac{1}{2 \pi \sigma_{\perp}^{2}} \exp \left[-\frac{\left(x-x^{i}\right)^{2}+\left(y-y^{i}\right)^{2}}{2 \sigma_{\perp}^{2}}-\frac{\left(\eta_{\mathrm{s}}-\eta_{\mathrm{s}}^{i}\right)^{2}}{2 \sigma_{\eta}^{2}}\right]
$$

Here $x$ and $y$ are transverse coordinates and $\eta_{\mathrm{s}}$ is space-time rapidity. Each particle $i$ is supposed to be produced at $\left(x^{i}, y^{i}\right)$ in the transverse plane and $\eta_{\mathrm{s}}^{i}(=Y)$ in the longitudinal direction. $\sigma_{\perp}$ and $\sigma_{\eta}$ are smearing parameters in the Gaussian function and $K$ is an overall normalization. We set $\sigma_{\perp}=0.3 \mathrm{fm}$, $\sigma_{\eta}=0.3, \tau_{0}=0.6 \mathrm{fm} / c$ and $K=5.6$ and use this parameter set throughout this study.

Figure 1 shows an example of transverse $\left(\eta_{\mathrm{s}}=0\right)$ and longitudinal $(x=0 \mathrm{fm})$ profiles of the initial entropy density in $p+\mathrm{Au}, d+\mathrm{Au}$ and ${ }^{3} \mathrm{He}+\mathrm{Au}$ collisions. The impact parameter is chosen to be $b=$ $0 \mathrm{fm}$. One sees differences of the numbers of separated hot spots which correspond to transverse positions of nucleons in colliding $p, d$ and ${ }^{3} \mathrm{He}$. In the longitudinal plane, the entropy density is distributed mainly in the negative rapidity region, i.e., Au-going direction. In all cases, initial entropy density has a bumpy structure in both a transverse and longitudinal plane.

\section{Results}

Figure 2 shows multiplicity distributions in negative rapidity region $(-3.9<\eta<-3.0)$ in $p+\mathrm{Au}$, $d+\mathrm{Au}$ and ${ }^{3} \mathrm{He}+\mathrm{Au}$ collisions at $\sqrt{s_{\mathrm{NN}}}=200 \mathrm{GeV}$. Since we employed, for the initial conditions, 

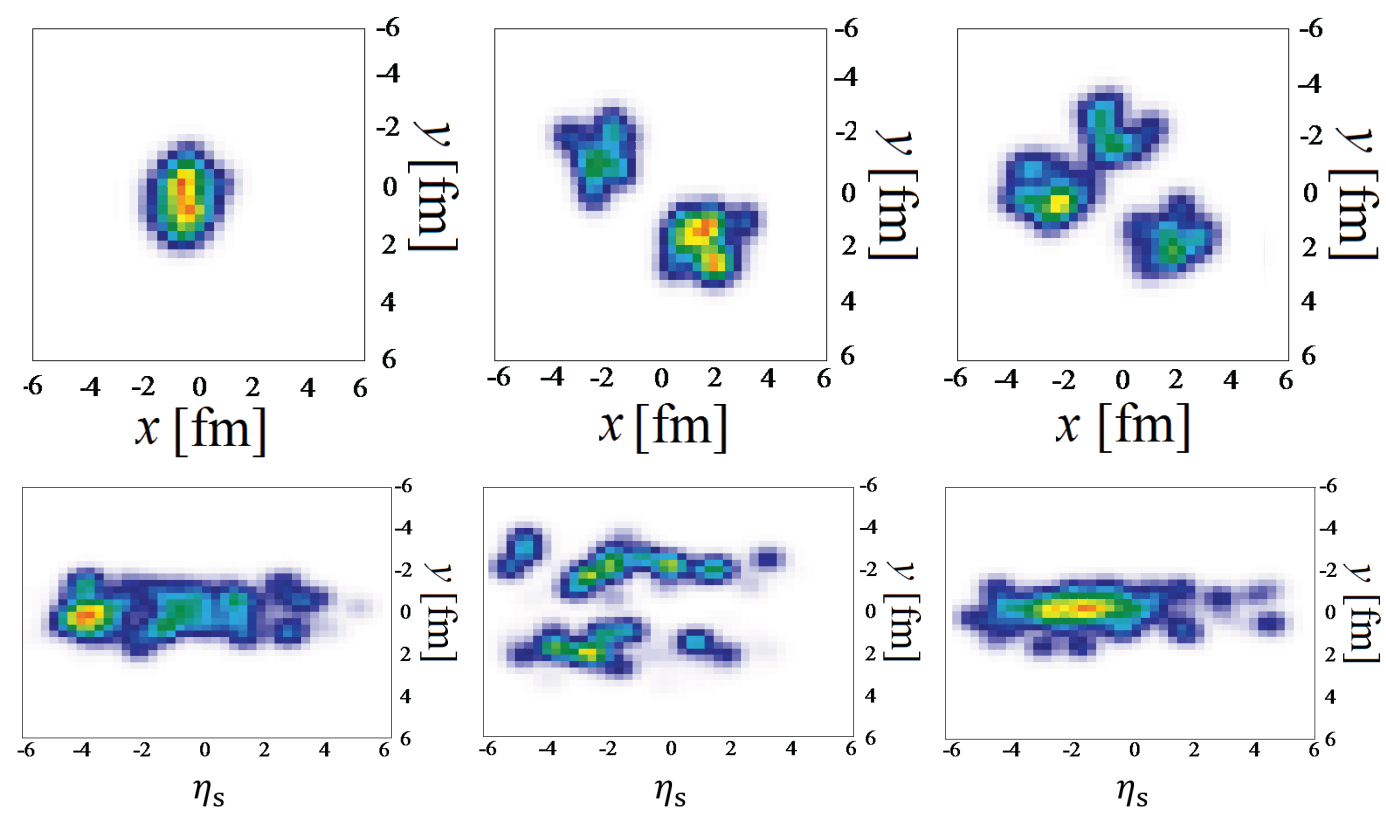

Figure 1. Examples of initial entropy density profile in the transverse plane (upper) and in the longitudinal plane (lower) in $p+\mathrm{Au}$ (left), $d+\mathrm{Au}$ (middle) and ${ }^{3} \mathrm{He}+\mathrm{Au}$ (right) collisions at $\sqrt{s_{\mathrm{NN}}}=200 \mathrm{GeV}$.

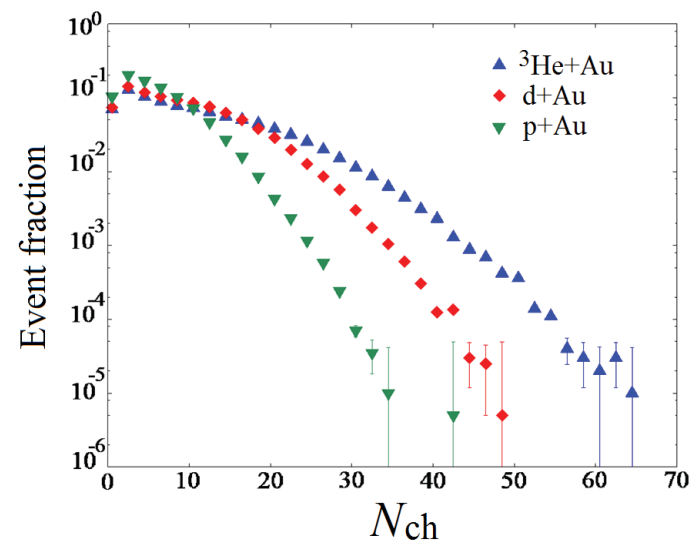

Figure 2. Multiplicity distributions of charged hadrons in $-3.9<\eta<-3.0$ in $p+\mathrm{Au}$ (green), $d+\mathrm{Au}$ (red) and ${ }^{3} \mathrm{He}+\mathrm{Au}$ (blue) collisions at $\sqrt{s_{\mathrm{NN}}}=200 \mathrm{GeV}$. 

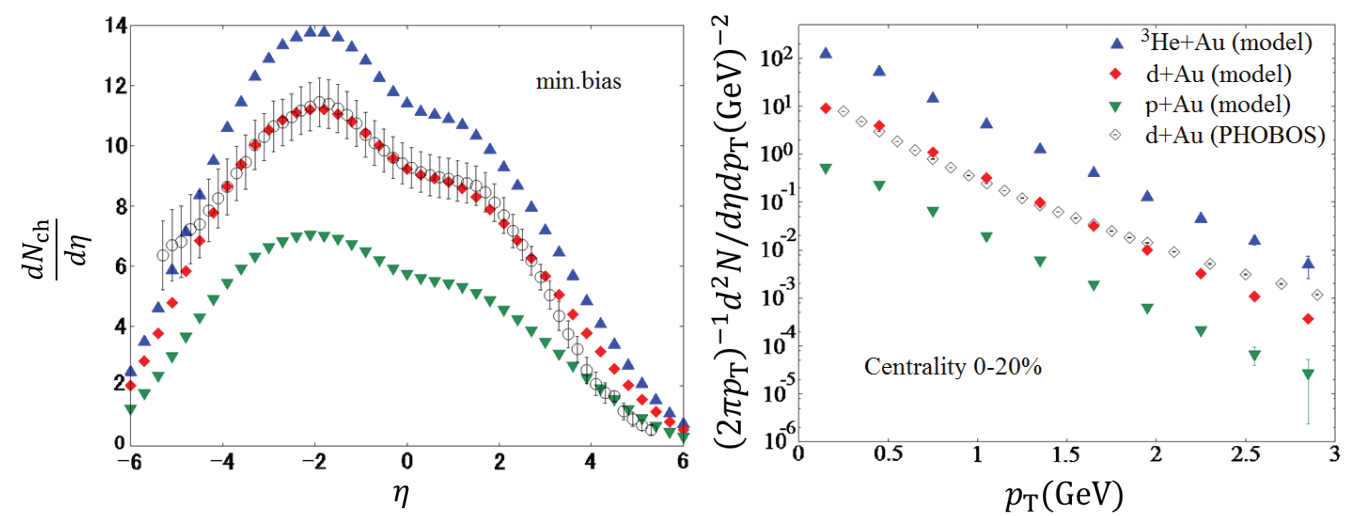

Figure 3. Pseudorapidity distributions in minimum bias (left) and transverse momentum distributions in $0.2<$ $\eta<1.4$ in central $0-20 \%$ events (right). Results in $p+\mathrm{Au}$ (green), $d+\mathrm{Au}$ (red) and ${ }^{3} \mathrm{He}+\mathrm{Au}$ (blue) collisions at $\sqrt{s_{\mathrm{NN}}}=200 \mathrm{GeV}$ are compared with the PHOBOS data (black) $[13,14]$. To see the plots clearly, the result in ${ }^{3} \mathrm{He}+\mathrm{Au}(p+\mathrm{Au})$ collisions is multiplied (divided) by ten in the right figure.

PYTHIA in which multiplicities fluctuate in $p+p$ collisions, multiplicity distributions in small colliding systems take over these fluctuations. These multiplicity distributions are used for a later centrality cut.

Figure 3 shows the pseudorapidity distributions (left) and transverse momentum $\left(p_{\mathrm{T}}\right)$ distributions (right) of charged hadrons in $p+\mathrm{Au}, d+\mathrm{Au}$ and ${ }^{3} \mathrm{He}+\mathrm{Au}$ collisions at $\sqrt{s_{\mathrm{NN}}}=200 \mathrm{GeV}$. Pseudorapidity distributions are averaged over minimum bias events, while $p_{\mathrm{T}}$ distributions are in $0-20 \%$ centrality. The PHOBOS data $[13,14]$ in $d+\mathrm{Au}$ collisions are also shown for comparison. We reproduce the shape and yield of the pseudorapidity distribution in $d+\mathrm{Au}$ collisions and find the idea of initial "rapidity triangle/trapezoid" works well. The $p_{\mathrm{T}}$ slope in Fig. 3 (left) is consistent with the PHOBOS data in the low $p_{\mathrm{T}}$ region. However, our result deviates from the PHOBOS data in high $p_{\mathrm{T}}$ region since we do not have any jet components in our model.

We analyse the transverse momentum dependence of the elliptic flow parameter $v_{2}$ of charged hadrons in $0-5 \%$ central ${ }^{3} \mathrm{He}+\mathrm{Au}$ collisions at $\sqrt{s_{\mathrm{NN}}}=200 \mathrm{GeV}$ in Fig. 4. The PHENIX data are also shown for comparison. In both analyses, an event plane method is used to obtain the elliptic flow parameter $v_{2}$. We reasonably reproduce the PHENIX $v_{2}$ data [3] from the integrated dynamical model. This indicates the QGP fluid + hadronic gas picture works well also in this small colliding system.

Pseudorapidity and centrality dependences of elliptic flow parameters are shown in Fig. 5. To see how much elliptic flow is generated in the QGP fluid stage, the results with hadronic rescatterings switched off are shown $[15,16]$. These elliptic flow parameters are obtained by using the two particle cumulant method. In Fig. 5 (left), the shape of elliptic flow is asymmetric with respect to pseudorapidity. Elliptic flow in the Au-going side (in the left-hand side) is larger than that in the ${ }^{3} \mathrm{He}$-going side (in the right-hand side) due to a difference of multiplicities as shown in Fig. 3 (left). In Fig. 5 (right), top $10 \%$ results, $10-20 \%$ results and so on are shown from right to left. Our results show elliptic flow parameters increase with the multiplicity. In both figures, roughly $90 \%$ of final $v_{2}$ is generated in the QGP fluid stage. The situation is quite similar to that in Au+Au collisions at the RHIC energy. This indicates that a large fraction of $v_{2}$ is generated in the QGP fluid state even in small colliding systems. 


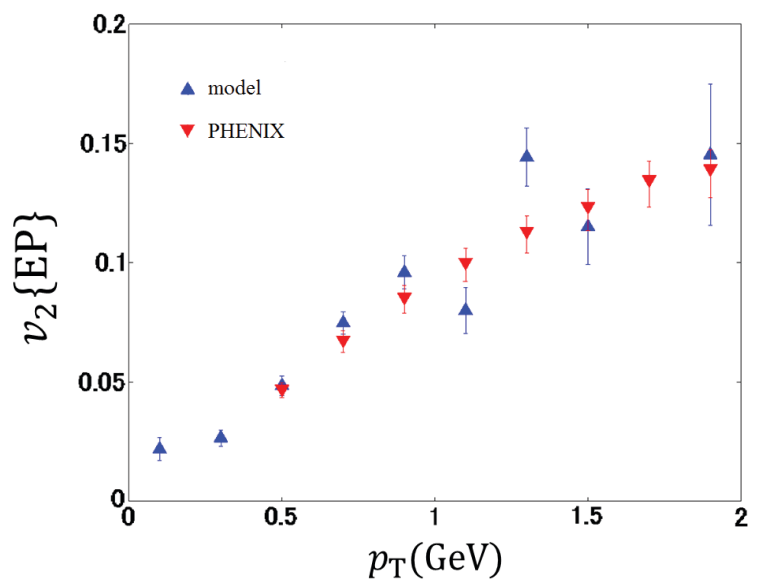

Figure 4. Preliminary result of $v_{2}\left(p_{\mathrm{T}}\right)$ of charged hadrons at midrapidity $(|\eta|<0.35)$ in $0-5 \%$ central ${ }^{3} \mathrm{He}+\mathrm{Au}$ collisions at $\sqrt{s_{\mathrm{NN}}}=200 \mathrm{GeV}$. Our results (blue) are compared with the PHENIX data (red) [3].
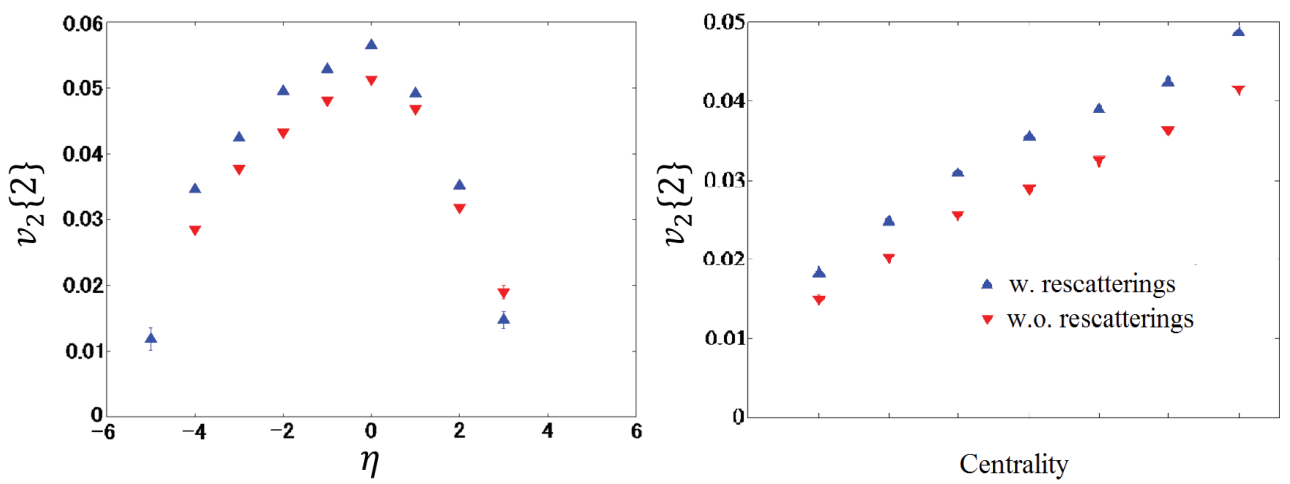

Figure 5. Pseudorapidity dependence of elliptic flow (left) and centrality dependence of elliptic flow (right) in ${ }^{3} \mathrm{He}+\mathrm{Au}$ collisions at $\sqrt{s_{\mathrm{NN}}}=200 \mathrm{GeV}$. In the right figure, from right to left, results in 0-10\%, 10-20\%, 20-30\%, $30-40 \%, 40-50 \%, 50-60 \%$ and $60-70 \%$ centrality are shown. Blue (Red) plots are results with (without) hadronic rescatterings.

\section{Summary}

We studied flow observables in small colliding systems by employing an integrated dynamical model. We extended a model of initial conditions to describe dynamics in small colliding systems by taking account of event-by-event fluctuations of geometry and entropy densities. Using the newly developed version of the model, we analysed multiplicity, pseudorapidity distributions and $p_{\mathrm{T}}$ spectra as well as $p_{\mathrm{T}}$-/pseudorapidity-/centrality-dependence of elliptic flow in small colliding systems. From these results, we found that the observed large $v_{2}$ can be attributed to both QGP expansion and hadronic 
rescatterings, but a large fraction of $v_{2}$ is generated in the QGP fluid stage even in small colliding systems.

\section{References}

[1] "RHIC Scientists Serve Up Perfect Liquid", http://www.bnl.gov/newsroom/news.php?a=1303

[2] J.-Y. Ollitrault, Phys. Rev. D46, 229 (1992)

[3] A. Adare et al. (PHENIX), Phys. Rev. Lett. 444, 212301 (2013)

[4] A. Adare et al. (PHENIX), Phys. Rev. Lett. 115, 142301 (2015)

[5] A. Adare et al. (PHENIX), Phys. Rev. Lett. 114, 192301 (2015)

[6] T. Hirano, P. Huovinen, K. Murase and Y. Nara, Prog. Part. Nucl. Phys. 70, 108 (2013)

[7] F. Cooper and G. Frye, Phys. Rev. D10, 186 (1974)

[8] Y. Nara, N. Otuka, A. Ohnishi, K. Niita and S. Chiba, Phys. Rev. C61, 024901 (2000)

[9] T. Sjöstrand et al., Comput. Phys. Commun. 191, 159(2015)

[10] A. Adil and M. Gyulassy, Phys. Rev. C72, 034907 (2005)

[11] S.J. Brodsky, J.F. Gunion and J.H. Kuhn, Phys. Rev. Lett. 39, 1120 (1977)

[12] T. Hirano, U.W. Heinz, D. Kharzeev, R. Lacey and Y. Nara, Phys. Lett. B636, 299 (2006)

[13] B.B. Back et al. (PHOBOS), Phys. Rev. C72, 031901 (2005)

[14] B.B. Back et al. (PHOBOS), Phys. Rev. Lett. 91, 072302 (2003)

[15] S. Takeuchi, K. Murase, T. Hirano, P. Huovinen and Y. Nara, Phys. Rev. C92, 044907 (2015)

[16] T. Hirano, U.W. Heinz, D. Kharzeev, R. Lacey and Y. Nara, Phys. Rev. C77, 044909 (2008) 